\title{
Isolated coronal urethral duplication in an adolescent presenting with enuresis
}

\author{
AA Bokhari, 1,2,3,4 ID Sookram, ${ }^{1,2,3}$ (D) A Adam ${ }^{1,2,3}$ iD \\ ${ }^{1}$ Department of Urology, Helen Joseph Hospital, South Africa \\ ${ }^{2}$ Department of Paediatric Urology, Rahima Moosa Mother \& Child (Coronation) Hospital, South Africa \\ ${ }^{3}$ Division of Urology, Department of Surgery, School of Clinical Medicine, Faculty of Health Sciences, University of the Witwatersrand, \\ South Africa \\ ${ }^{4}$ Department of Urology, Hail University, Saudi Arabia
}

Corresponding author, email: Akram.0553@hotmail.com

\begin{abstract}
Summary
This report describes an isolated urethral duplication in the coronal plane in a child referred with primary 'enuresis'. This presentation is unique because duplications usually occur in the sagittal plane. In patients with suspected urethral duplication, magnetic resonance imaging in conjunction with catheterisation of the distal duplicate opening accurately delineates the abnormality, so that individualised treatment strategies can be considered.

Keywords: urethra, duplication, coronal, childhood, enuresis
\end{abstract}

\section{Case report}

A 15-year-old male presented with a history of post micturition dribbling and 'enuresis'. He had no medical comorbidity nor any erectile dysfunction. His referral letter incorrectly assessed him as having a normal examination. However, on penile examination, a second urethral meatal opening $10 \mathrm{~mm}$ lateral to the meatus on the glans was observed (Figure 1A). No other abnormality was found on examination. A voiding cystourethrogram (VCUG) was performed, which demonstrated a double urethra exiting from the membranous urethra (Figure 1B).

This anomaly was also defined using pelvic magnetic resonance imaging (MRI). For better definition of the duplicate urethra, prior to MRI the duplicate urethral opening was cannulated with a ureteric catheter (Axxcess ${ }^{\mathrm{TM}}$ Catheter, Boston Scientific, Marlborough, MA 01752-1234, USA).

For stability and orientation of the penis during the study, the glans and penile shaft were secured in the midline to the lower abdominal wall, using an adhesive dressing.

The MRI study confirmed a urethral duplication, within the right corporal body, in the coronal plane (Figure 2A). On cystoscopy the urethral ostium was seen, and a guide wire was advanced through the ostium of the duplicate urethra, exiting from the duplicate urethral meatus (Figure 2B). Despite adequate counselling, the patient
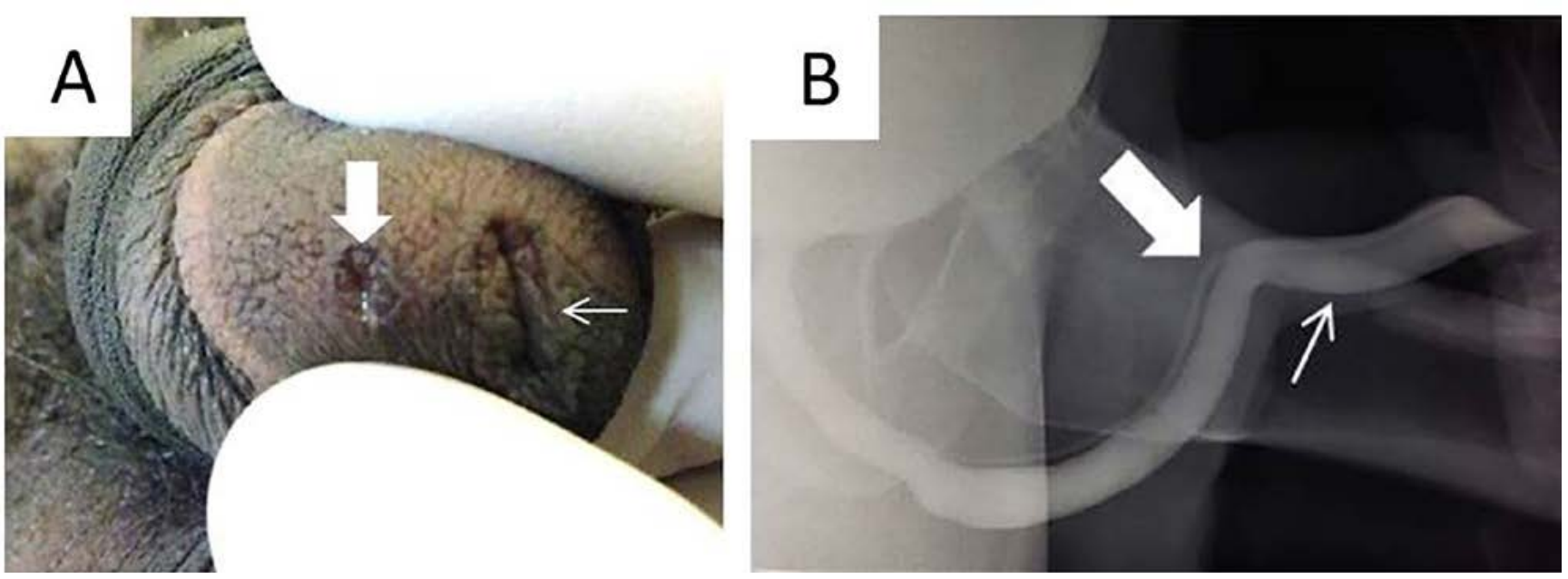

Figure 1A: Clinical photograph of the glans penis. The duplicate urethral meatus (thick arrow) is visualised on the right lateral coronal plane to the urethra (thin arrow)

Figure 1B: Voiding Cysto Urethrogram (VCUG) depicting the duplicate urethra (thick arrow) in relation to the orthotopic urethra (thin arrow) 


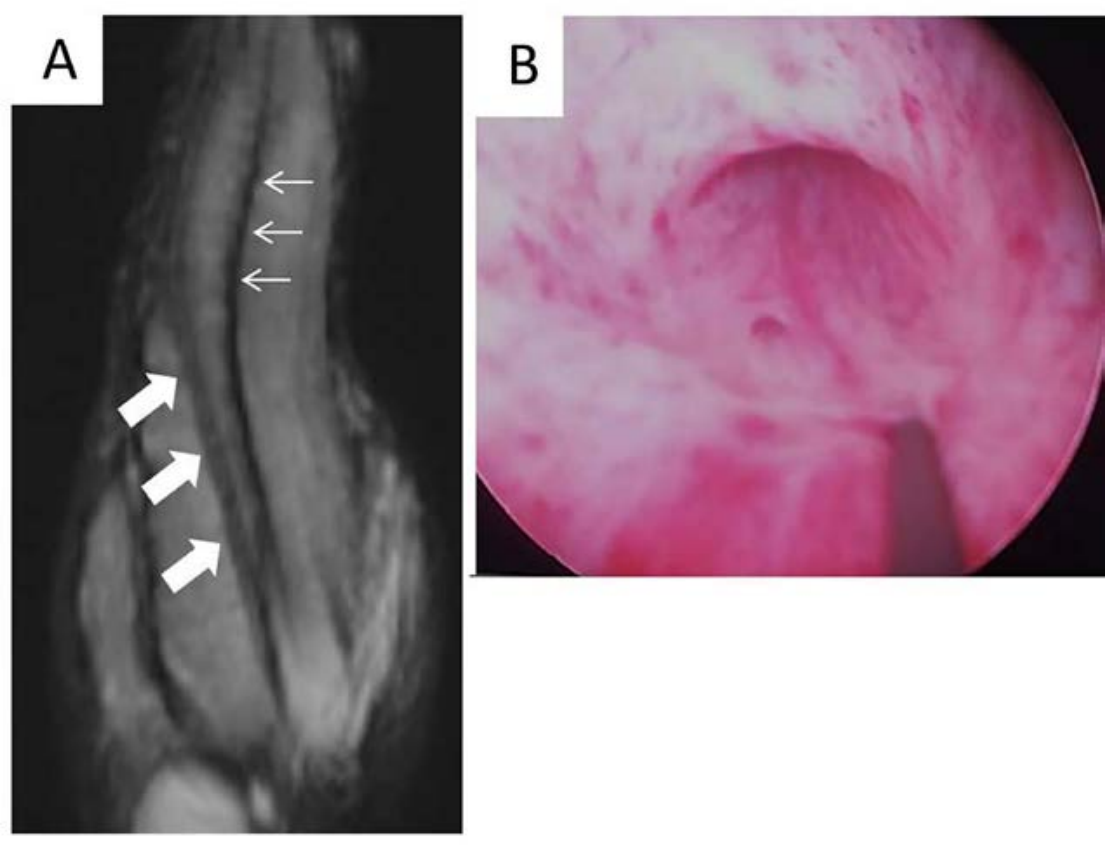

Figure 2A: MRI study (T2 weighted image) of the penile shaft (longitudinal section). The duplicate urethra (thick arrows) is visualised, within the right corpora body, in the right lateral coronal plane to the urethra (thin arrows). Images have been taken with the penis placed in the midline against the anterior abdominal wall to allow adequate visualisation of the duplicate urethra

Figure 2B: Endoscopic view at cystoscopy, showing a guidewire entering the urethral ostium of the duplicate urethra

and family declined definitive surgical repair, endoscopic ablation or injectable therapy for the duplicated urethra and no complications were observed in long-term follow-up of the patient.

\section{Discussion}

Congenital urethral duplication in general is a rare congenital anomaly, with less than 200 cases reported in the literature. ${ }^{1}$ Duplication in the coronal plane was first described in 2000 by Salle et al. ${ }^{1}$

During the evaluation of patients with urethral duplication, it is advocated to exclude other anomalies and complications such as vesico-ureteric reflex or genitourinary infection. ${ }^{2}$ This is the third reported case of isolated urethral duplication in the coronal plane in male childhood with no other associated anomalies reported in the literature. ${ }^{3,4}$

Both previous isolated coronal duplication cases in the pediatric cohort were diagnosed under a year of age, 3,4 one of them underwent surgical intervention. ${ }^{4}$ The precise embryology of the entity of urethral duplication remains undefined. ${ }^{5}$

Several authors attempted to classify urethral duplication over the decades and the most commonly used classification being that described by Effman et al. ${ }^{6}$

They classified urethral duplication into 3 types: Type I - incomplete urethral duplication (A. Distal, B. Proximal), Type II - complete urethral duplication (A. 2 meatus, B. 1 meatus) and Type III - urethral duplication as a component of partial or complete caudal duplication. ${ }^{6}$

Since coronal plane duplication is largely undefined according to the commonest classification system of Effman et al. ${ }^{6}$ various newer classification systems attempting to incorporate more recently described abnormalities associated with urethral duplication have been proposed. ${ }^{7,8}$
The diagnosis of urethral duplication is usually established on imaging, most commonly the VCUG study with a separate VCUG of both urethral openings assists to confirm cases of complete duplication. $^{2}$ MRI is another excellent investigation modality for the evaluation of urethral duplication, as well as other associated genitourinary abnormalities. ${ }^{9}$ As illustrated with this case, the MRI can be used with cannulation of accessory urethra, this has also previously been shown to enhance imaging by allowing for distention of the duplicate urethra. ${ }^{9}$

The treatment options offered include conservative or surgical intervention depending on the individual case and the related anatomical variation observed. ${ }^{2}$ Surgery should be considered in genitourinary anomalies because of the disturbing symptoms of outlet obstruction, urinary incontinence and double stream. ${ }^{9}$ Recently, treatment specific classifications have also been proposed in this regard. ${ }^{1}$ The target for treatment of these patients is to restore kidney function, continence and cosmetic option. $^{7}$ Our recommendation for this patient would have been endoscopic ablation and injectable closure, due to the tract's proximity with the corporal bodies. Disturbance of the glans, meatal stenosis, urethral stricture or repair dehiscence urinary tract infection and erectile dysfunction are known complications, but in general the majority of patients have a good outcome. . $^{5,8}$

\section{Conclusion}

The first report of unclassified, isolated coronal urethral duplication in a male adolescent with suspected 'enuresis' is presented. Careful examination of the glans and meatus is an essential component of the physical examination in all male children and adolescents presenting with voiding dysfunction or enuresis. Identification will then allow appropriate imaging to delineate the abnormality and discuss treatment options.

\section{Acknowledgements}

We wish to acknowledge the kind assistance with the image retrieval and literature search from Dr Kalli Spencer (Division of Urology, University of the Witwatersrand) and Dr Vladimir Orlov (Regional Children's Hospital, Rostovon-Don, Russia).

\section{Conflict of interest}

The authors declare no real or apparent conflicts of interest.

\section{Funding source}

None. 


\section{Ethical approval}

Local institutional ethics approval (Human Research Ethics Committee) Certificate M.160394 for publication of this report had been attained.

\section{ORCID}

AA Bokhari (D) https://orcid.org/0000-0002-1609-836X

J Sookram (D) https://orcid.org/0000-0002-3911-8511

A Adam (D) https://orcid.org/0000-0001-9069-3282

\section{REFERENCES}

1. Salle J, Sibai H, Rosenstein D, Brzezinski A, Corcos J. Urethral duplication in the male: review of 16 cases. J Urol. 2000;163:1936-40.

2. Berrocal T, Novak S, Arjonilla A, et al. Complete duplication of bladder and urethra in the coronal plane in a girl: case report and review of the literature. Pediatr Radiol. 1999;29(3):171-3.

3. Ching C, Palmer J. Collateral urethral duplication. Dialogues Pediatr Urol. 2008;29:16-7.
4. Frederick L, Benson AD. A case of coronal urethral duplication with no other abnormalities. Can J Urol [Internet]. 2011 Feb;18(1):5557-9.

5. Slavov C, Donkov I, Popov E. Case of duplication of the urethra in an adult male, presenting with symptoms of bladder outlet obstruction. Eur Urol. 2007;52(4):1249-51.

6. Effman E, Lebowitz R, Colodny A. Duplication of urethra. Radiology. 1976 Apr;119(1):179-85.

7. Abouzeid AA, Safoury HS, Mohammad SA, et al. The double urethra: revisiting the surgical classification. Ther Adv Urol. 2015;7(2):76-84.

8. Lima M, Destro F, Maffi M, Persichetti Proietti D, Ruggeri G. Practical and functional classification of the double urethra: a variable, complex and fascinating malformation observed in 20 patients. J Pediatr Urol. 2017 Feb;13(1):42.e1-42.e7.

9. Parashari U, Singh R, Kohli N, Bhadury S. MRI in congenital duplication of urethra. Indian J Radiol Imaging. 2009;19(3):232. 